\title{
Status of the KASCADE-Grande Experiment
}

\author{
K.-H. Kampert ${ }^{\mathrm{a}}$ b*, T. Antoni ${ }^{\mathrm{a}}$, W.D. Apel ${ }^{\mathrm{b}}$, F. Badea ${ }^{\mathrm{\dagger}}$, K. Bekk ${ }^{\mathrm{b}}$, A. Bercuci ${ }^{\mathrm{b}}{ }$, M. Bertaina $^{\mathrm{c}}$, \\ H. Blümer ${ }^{\text {a }}$, H. Bozdog ${ }^{\mathrm{b}}$, I.M. Brancus ${ }^{\mathrm{d}}$, C. Büttner ${ }^{\mathrm{a}}$, A. Chiavassa ${ }^{\mathrm{c}}$, K. Daumiller $^{\mathrm{a}}$, P. Doll ${ }^{\mathrm{b}}$, \\ J. Engler ${ }^{\mathrm{b}}$, F. Feßler ${ }^{\mathrm{a}}$, P.L. Ghia ${ }^{\mathrm{e}}$, H.J. Gils ${ }^{\mathrm{b}}$, R. Glasstetter ${ }^{\mathrm{a}}$, R. Haeusler ${ }^{\mathrm{a}}$, A. Haungs ${ }^{\mathrm{b}}$, D. Heck ${ }^{\mathrm{b}}$, \\ J.R. Hörandel ${ }^{\mathrm{a}}$, A. Iwan ${ }^{\mathrm{a} \ddagger}$, H.O. Klages ${ }^{\mathrm{b}}$, G. Maier ${ }^{\mathrm{b}}$, H.J. Mathes ${ }^{\mathrm{b}}$, H.J. Mayer ${ }^{\mathrm{b}}$, D. Martello ${ }^{\mathrm{b} \S}$, \\ J. Milke ${ }^{\mathrm{a}}$, C. Morello ${ }^{\mathrm{e}}$, M. Müller ${ }^{\mathrm{b}}$, G. Navarra ${ }^{\mathrm{c}}$, R. Obenland ${ }^{\mathrm{b}}$, J. Oehlschläger ${ }^{\mathrm{b}}$, S. Ostapchenko ${ }^{\text {ฯ }}$, \\ M. Petcu ${ }^{\mathrm{d}}$, H. Rebel ${ }^{\mathrm{b}}$, M. Roth $^{\mathrm{b}}$, H. Schieler ${ }^{\mathrm{b}}$, J. Scholz ${ }^{\mathrm{b}}$, T. Thouw ${ }^{\mathrm{b}}$, G.C. Trinchero ${ }^{\mathrm{e}}$, H. Ulrich $^{\mathrm{a}}$, \\ R. Ulrich ${ }^{\mathrm{a}}$, J.H. Weber ${ }^{\mathrm{a}}$, A. Weindl ${ }^{\mathrm{b}}$, J. Wentz ${ }^{\mathrm{b}}$, J. Wochele ${ }^{\mathrm{b}}$, J. Zabierowski ${ }^{\mathrm{f}}$, and S. Zagromski ${ }^{\mathrm{b}}$ \\ ${ }^{a}$ University of Karlsruhe, Institut für Kernphysik, P.O. Box 3640, 76021 Karlsruhe, Germany \\ ${ }^{\text {b} I n s t i t u t ~ f u ̈ r ~ K e r n p h y s i k, ~ F o r s c h u n g s z e n t r u m ~ K a r l s r u h e, ~} 76021$ Karlsruhe, Germany \\ ${ }^{\text {c} D i p a r t i m e n t o ~ d i ~ F i s i c a ~ G e n e r a l e ~ d e l l ' U n i v e r s i t a ̀, ~} 10125$ Torino, Italy \\ d National Institute of Physics and Nuclear Engineering, 7690 Bucharest, Romania \\ e Istituto di Fisica dello Spazio Interplanetario del CNR, Sezione di Torino, 10133 Torino, Italy \\ ${ }^{\mathrm{f}}$ Soltan Institute for Nuclear Studies, 90950 Lodz, Poland
}

The status and capabilities of the KASCADE-Grande extensive air shower experiment are presented. The installation is located at Forschungszentrum Karlsruhe and comprises a large collecting area $\left(0.5 \mathrm{~km}^{2}\right) \mathrm{electro-}^{-}$ magnetic array (Grande) operated jointly with the existing KASCADE detectors. KASCADE-Grande will cover the primary energy range $10^{16} \mathrm{eV}<E_{0}<10^{18} \mathrm{eV}$ overlapping with KASCADE around $10^{16} \mathrm{eV}$, thus providing continuous information on the primary energy and mass of cosmic rays from $3 \cdot 10^{14} \mathrm{eV}$ up to $10^{18} \mathrm{eV}$. The major goal of the measurements is the unambiguous observation of the "iron knee" expected in the cosmic ray spectrum at $E_{k}^{F e} \approx 10^{17} \mathrm{eV}$.

\section{INTRODUCTION}

The origin and acceleration mechanism of ultra-high energy cosmic rays $\left(E \gtrsim 10^{14} \mathrm{eV}\right)$ and the origin of the 'knee' at approx. $3 \cdot 10^{15} \mathrm{eV}$ have been subject to debate for several decades. Most commonly, it is assumed that cosmic rays gain their energy by first order Fermi acceleration at highly supersonic shock waves originating in supernova explosions. Simple dimensional estimates show that this process is limited to $E_{\max } \lesssim Z \times(\rho \times B)$, with $Z$ being the atomic number of the cosmic ray (CR) isotope and $\rho, B$ the size and magnetic field strength of the acceleration region. A more detailed examination of the astrophysical parameters suggests an upper

\footnotetext{
${ }^{*}$ Corresponding author: Karl-Heinz.Kampert@ik.fzk.de ${ }^{\dagger}$ on leave of absence from ${ }^{\mathrm{d}}$

$\ddagger$ and University of Lodz, Poland

${ }^{\S}$ permanent address: University Lecce, Italy

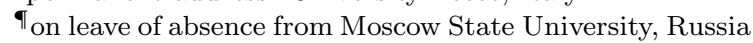

limit of acceleration of $E_{\max } \approx Z \times 10^{15} \mathrm{eV}$ [1.,2]. It is thus tempting to identify the knee with the maximum energy obtained by such accelerators. A brief compilation of alternative interpretations of the knee is given in Ref. 3. As a simple consequence of the aforementioned 'standard' picture, one expects to see a break at constant rigidity for all particles, i.e. protons would break off first and iron nuclei at correspondingly higher energy. This results in an increasingly heavier composition at energies above the knee.

Such a global change of composition is in fact observed by various experiments, including KASCADE [4.5] and EAS-TOP [6] (a recent compilation can be found in Ref. [7]). A rather convincing proof has recently been presented by the KASCADE collaboration 8.53 reporting preliminary evidence for a knee shifting towards higher energies with increasing mass of the primaries (see also contributions to this conference by $\mathrm{H}$. Ulrich et al. and M. Roth et al.). However, recon- 
struction properties and statistics do not allow to clearly identify the expected iron knee at $E \approx$ $10^{17} \mathrm{eV}$. The primary goal of the KASCADEGrande experiment is to uncover the possible existence of an iron break by providing high quality data from $10^{16}-10^{18} \mathrm{eV}$. The experiment covers an area of approx. $0.5 \mathrm{~km}^{2}$ and is located next to the KASCADE site in order to operate jointly with the existing KASCADE detectors. The combination with KASCADE will guarantee a cross calibration of the detectors and will provide full coverage from $3 \cdot 10^{14}$ to $10^{18} \mathrm{eV}$.

By the time of writing this article, all detector stations have been deployed and first showers were already observed with preliminary readout electronics. Full operation will start in January 2003 and full statistics will be reached after 3-4 years of data taking. The present status of the array, its resolutions and capabilities in reconstructing the average primary composition and its verification of the hadronic interaction models are discussed.

\section{EXPERIMENT}

A sketch of the KASCADE-Grande layout is presented in Fig. 1. It consists of three different detector arrays: KASCADE, Grande, and Piccolo.

The KASCADE experiment [9] comprises three major components: an array of electron and muon detectors, a central detector mainly for hadron measurements but with substantial muon detection areas, and an underground muon tracking detector (MTD). The $e / \gamma$ - and $\mu$-detectors cover an area of $200 \times 200 \mathrm{~m}^{2}$ and consist of 252 detector stations located on a square grid of 13 $\mathrm{m}$ separation providing in total about $490 \mathrm{~m}^{2}$ of $e / \gamma$ and $622 \mathrm{~m}^{2}$ of muon coverage. The detection thresholds for vertical incidence are $E_{e}>5 \mathrm{MeV}$ and $E_{\mu}>230 \mathrm{MeV}$. The central detector system $\left(320 \mathrm{~m}^{2}\right)$ consists of a highly-segmented hadronic calorimeter read out by 44,000 channels of warm liquid ionization chambers, a layer of scintillation counters above the shielding, a trigger plane of scintillation counters in the third layer and, at the very bottom, 2 layers of positional sensitive MWPC's and a streamer tube layer with pad

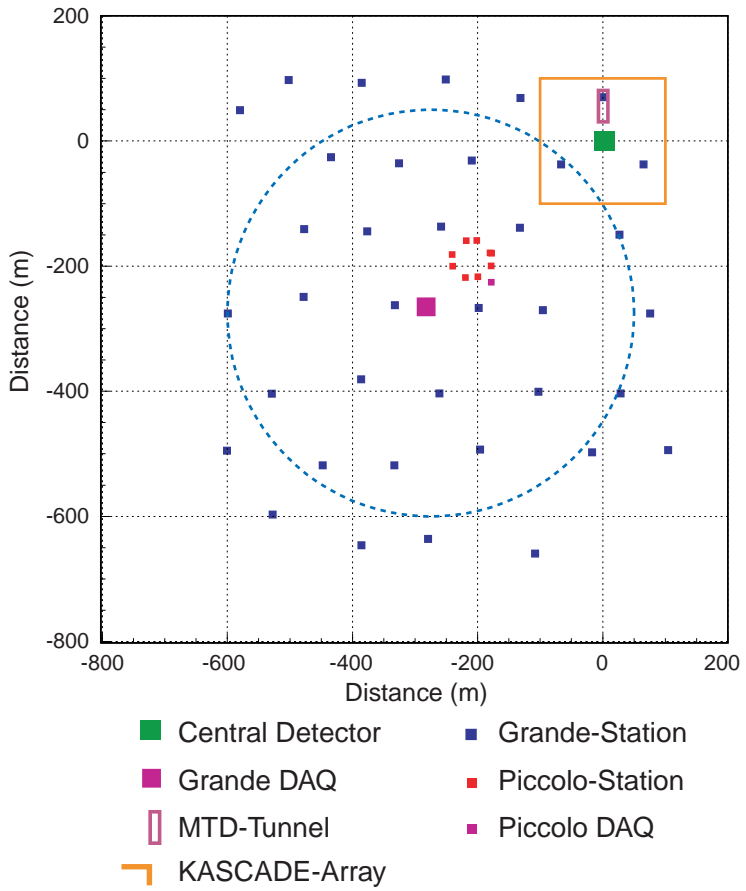

Figure 1. Layout of the KASCADE-Grande experiment. The circle indicates the acceptance region used in the analysis of Fig. 2.

read-out for muon tracking at $E_{\mu} \geq 2.4 \mathrm{GeV}$. The MTD is located in a $44 \times 5.4 \times 2.4 \mathrm{~m}^{3}$ tunnel, close to the central detector. It houses three horizontal and two vertical layers of positional sensitive limited streamer tubes for muon tracking at $E_{\mu} \geq 0.8 \mathrm{GeV}$. The accuracy in reconstructing the muon direction is $\sigma \approx 0.35^{\circ}$ [11]. With its approx. $1000 \mathrm{~m}^{2}$ of muon detector coverage KASCADE-Grande provides a much better muon sampling fraction (approx. $1000 \mathrm{~m}^{2}$ ) than any other previous experiment.

Grande comprises 37 stations of $10 \mathrm{~m}^{2}$ scintillation counters reused from the former EAS-TOP experiment [10]. The stations are positioned at an average mutual distance of approx. $130 \mathrm{~m}$. They are subdivided into 16 individual scintillation detectors $\left(80 \times 80 \mathrm{~cm}^{2}\right)$ read out by one phototube each. The inner four scintillation detectors are independently read out by low gain phototubes 


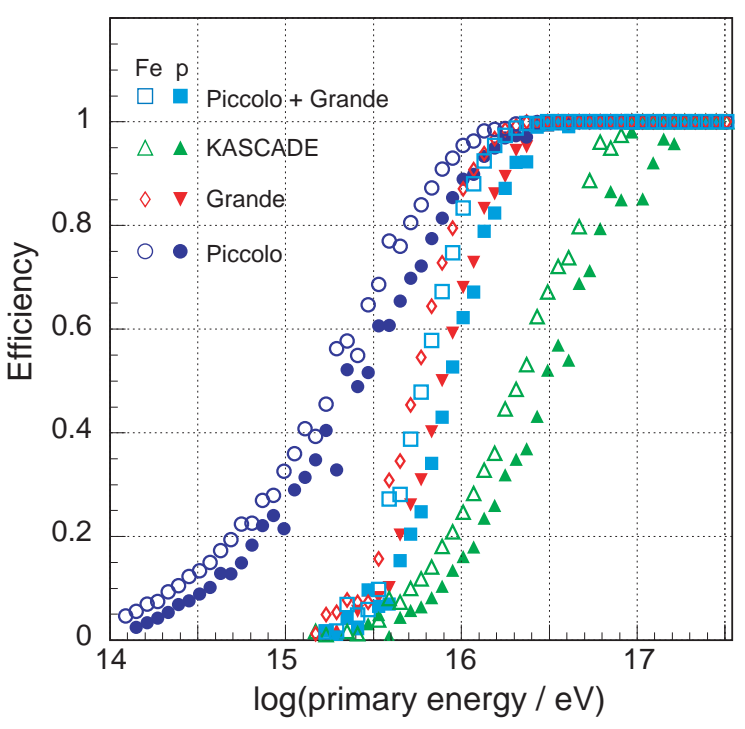

Figure 2. Simulated trigger efficiency for KASCADE-Grande. The up-triangles show the efficiency of the KASCADE array to trigger also the readout of Grande, the down-triangles and diamonds the efficiency of Grande (7-fold) only, the boxes show the efficiency that Piccolo is triggered together with Grande, and the circles show the efficiency for Piccolo to be triggered by showers in the acceptance region of Fig. 囵.

in order to provide a high dynamic range with high quality particle density and timing measurements from $\approx 0.3$ to $\approx 30000$ charged particles per $10 \mathrm{~m}^{2} 12$.

Piccolo consists of an array of 8 stations, each equipped with 12 scintillator plates (10 $\mathrm{m}^{2} /$ station) that were reused from the KARMEN neutrino experiment 13. The small array is placed between the centres of the KASCADE and Grande arrays and its main task is to provide a fast external trigger to Grande and KASCADE allowing to record coincident events with all the detectors of KASCADE-Grande. The simulated trigger efficiency using CORSIKA/QGSJET calculations is shown in Fig. 2 2 together with the trigger efficiency of the Grande array only. Using Piccolo, full detection efficiency is thus reached for $E \gtrsim 10^{16} \mathrm{eV}$ while in its absence, the KAS-
CADE multidetector information would only be availabe for showers above energies of $10^{17} \mathrm{eV}$. A summary of the detector components together with their most relevant parameters is given in Table 1 .

For triggering, Grande is electronically subdivided into 18 hexagonal clusters of 6 outer and one central station each. Requiring e.g. 4-fold coincidences within each cluster (demanding a signal over threshold in the central and in 3 adjacent outer stations) we measure a trigger rate of $\sim 0.3 \mathrm{~Hz}$ per cluster and a total trigger rate of Grande of $\sim 5.9 \mathrm{~Hz}$, well in expectation with simulation results. To improve the signal/noise ratio for low particle densities, the PMT signals will be amplified and shaped in the Grande stations. Digitisation is done after transmission via $700 \mathrm{~m}$ long shielded cables in peak sensing ADCs located in the central Grande-DAQ station. All detector components are deployed and data taking will start in January 2003.

\section{ANALYSIS}

After three years of data taking the total exposure of KASCADE-Grande will be $\Gamma \simeq 10^{14}$ $\mathrm{m}^{2} \mathrm{sr}$ s. This corresponds to the following numbers of collected events (including the trigger efficiency of Piccolo): $n\left(>10^{16} \mathrm{eV}\right) \simeq 1.7 \cdot 10^{6}$, $n\left(>10^{17} \mathrm{eV}\right) \simeq 2.5 \cdot 10^{4}, n\left(>10^{18} \mathrm{eV}\right) \simeq 250$. KASCADE-Grande will thus provide statistically significant physical information up to about $10^{18} \mathrm{eV}$.

The basic experimental observables will be: (i) the muon density at core distances between 300 and $600 \mathrm{~m}$ providing, after applying a fit to the observed muon lateral distribution, the muon density at $600 \mathrm{~m}, \rho_{\mu 600}$, (ii) the muon production heights $\left(h_{\mu}\right)$ reconstructed from the KASCADE muon tracking detector by means of triangulation (including timing information for the muons in the KASCADE central detector), and (iii) the shower size $\left(N_{c h}\right)$ and lateral electron density profile from the extended electromagnetic (e.m.) Grande array.

The reconstruction accuracies of the Grande array have been estimated by CORSIKA-QGSJET simulations. For the energy range of interest 


\begin{tabular}{cccccc}
\hline Detector & \# of Channels & Spacing $(\mathrm{m})$ & Total Area $\left(m^{2}\right)$ & Threshold $E_{k i n}$ & Particle Type \\
\hline Array $e / \gamma$ & 252 & 13 & 490 & $5 \mathrm{MeV}$ & $e$ \\
Array $\mu$ & 192 & 13 & 622 & $230 \mathrm{MeV} \times \sec \theta$ & $\mu$ \\
Trigger Plane & 456 & - & 208 & $490 \mathrm{MeV} \times \sec \theta$ & $\mu$ \\
MWPCs & 26080 & - & 129 & $2.4 \mathrm{GeV} \times \sec \theta$ & $\mu$ \\
Calorimeter & 44000 & - & 304 & $50 \mathrm{GeV}$ & Hadrons \\
Muon Tunnel & 24576 & - & 128 & $800 \mathrm{MeV}$ & $\mu$ \\
Grande & 74 & $\langle 130\rangle$ & 370 & $3 \mathrm{MeV}$ & $e, \mu$ \\
Piccolo & 16 & $\langle 25\rangle$ & 80 & $5 \mathrm{MeV}$ & $e, \mu$ \\
\hline
\end{tabular}

Table 1

Compilation of detector components and their particle detection thresholds.

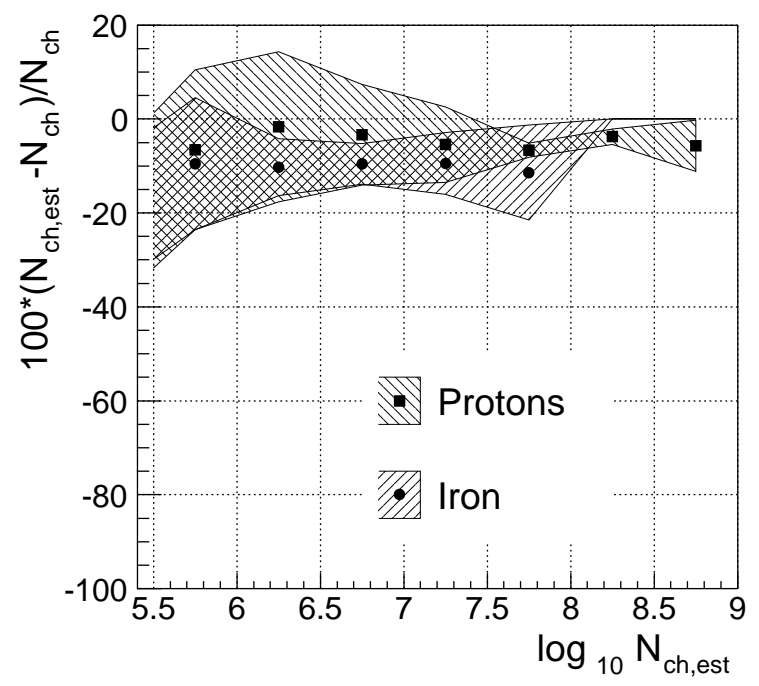

Figure 3. Simulated resolution of the reconstructed shower size in Grande for proton and iron induced primaries using CORSIKAQGSJET simulations and requiring 7-fold coincidences.

$\left(N_{c h} \geq 10^{7} ; E \gtrsim 10^{16} \mathrm{eV}\right.$, vertical showers $)$ the shower core position can be reconstructed to better than $5 \mathrm{~m}$ and the shower direction to better than $1.5^{\circ}$. Using not yet optimized lateral distribution functions, the shower size can be reconstructed to better than $10 \%$ (see Fig. 3).

Typical muon densities for $E_{0}=10^{17} \mathrm{eV}$ protons will be $\rho_{\mu 600} \approx 0.1 \mathrm{~m}^{-2}$. Considering a detection area of about $800 \mathrm{~m}^{2}$ for low energy

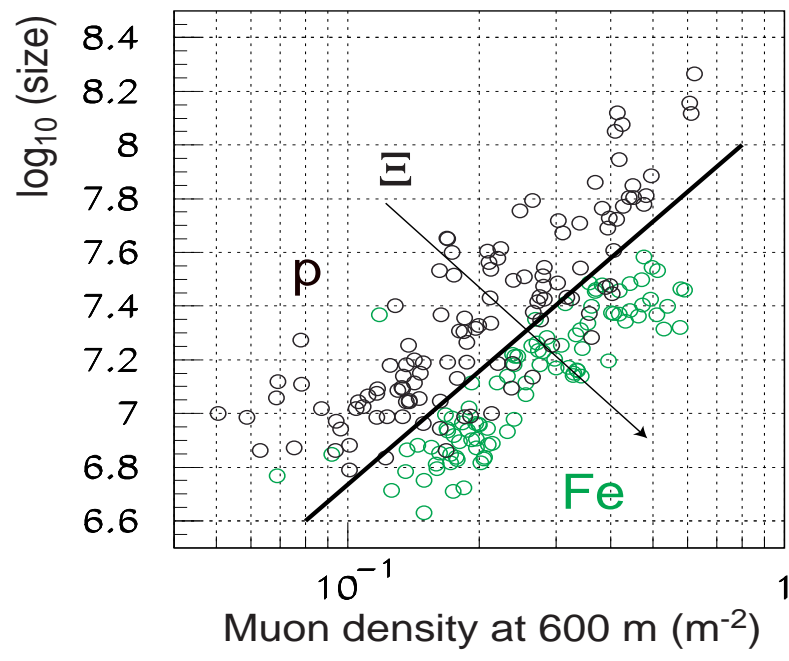

Figure 4. QGSJET Simulations of proton and iron primaries in the energy range of $2 \cdot 10^{16} \leq$ $E \leq 5 \cdot 10^{17} \mathrm{eV}$. The light symbols represent iron and the dark ones proton simulations. Both, shower fluctuations and experimental uncertainties are taken into account. The $\Xi$ line is used as a guideline for the $\Xi$ mass axis.

muons, we expect an average of 80 muons detected on a event by event basis meaning a statistical fluctuation of about $12 \%$.

Monte Carlo simulations using the CORSIKA package have been performed also to check the experiment capabilities for testing the hadronic interaction models and reconstructing the mass of primary CRs through the three observables $N_{e}$, 
$\rho_{\mu 600}$, and $h_{\mu}$. An example is presented in Fig. 1 . Here, a $N_{e}$ vs $\rho_{\mu 600}$ scatter plot is shown for proton and iron induced simulated air showers. The primary composition is simply defined in terms of the mass parameter $\Xi$, whose definition through $N_{e}$ and $\rho_{\mu 600}$ is shown in the same figure. The line separating $\mathrm{p}$ and $\mathrm{Fe}$ primaries best is indicated by the bold line. The mass axis $\Xi$ is thus shown orthogonal to this line. The parameter $\rho_{\mu 600}$ can, as first approximation, be used as energy estimator. It has been verified that the composition can be reconstructed in a reliable way even by such simple methods. However, the later analysis will use more advanced techniques, similar to those of Refs. [8, 14].

Hadronic interaction models will be studied by their differences seen in the longitudinal shower developments. Experimentally, this will be performed by the observed average relation between $h_{\mu}$ and $\rho_{\mu 600}$ and, to some extend, by studying hadronic observables using the KASCADE calorimeter.

\section{SUMMARY AND CONCLUSIONS}

KASCADE-Grande has been realized at the Forschungszentrum Karlsruhe by a joint operation of the KASCADE and EAS-TOP detectors. The apparatus is almost completed and data taking will start shortly. Within three years of effective data taking it will accumulate sufficient statistics up to $10^{18} \mathrm{eV}$. The experiment will cover an energy range that is only poorly known, essentially from old AKENO 15] and the lower tail of FLY'S EYE [16] data. Its task is to provide more information on the structure of the knee by testing the rigidity dependent model up to the energies of the "iron" group. Moreover, it will allow to test hadronic models in an energy range not accessible to present accelerators but important for CRs physics. Finally, it will provide a bridge to the measurement and interpretation of CRs for experiments working at much higher energies like the Pierre Auger, HIRES or EUSO projects.

\section{Acknowledgements}

The authors are indebted to the members of the engineering and technical staff of the KASCADE-
Grande collaboration, who contributed with enthusiasm and engagement to the construction of the experiment. The work has been supported by the Ministry for Research and Education of the Federal Government of Germany. Special thanks also go to INFN for allowing the use of the EAS-TOP equipment to build the Grande array. The collaborating Institute of Lodz is supported by the Polish State Committee for Scientific Research and the Institute of Bucharest by a grant of the Romanian National Academy for Science, Research and Technology. The KASCADEGrande work is embedded in the frame of scientific -technical cooperation (WTZ) projects between Germany and Romania, and between Germany and Poland.

\section{REFERENCES}

1. Drury L. O'C., Contemp. Phys. 35, 1994

2. Berezhko E.G. and Ksenofontov L.T., J. Exp. Theor. Phys. 89, (1999) 391.

3. Kampert K.-H. et al., Invited, Rapporteur, and Highlight papers of ICRC 2001, p240.

4. Glasstetter R. et al., Proc. 26th ICRC, HE2.2.03, 1999.

5. Antoni T. et al., Astropart. Phys. 16 (2002) 373.

6. Aglietta M. et al., Proc. 26th ICRC, HE2.2.05, 1999.

7. Swordy S. et al., Astropart. Physics 18 (2002) 129.

8. Ulrich H. et al., $27^{\text {th }}$ ICRC, Hamburg, (2001) 97.

9. Antoni T. et al., preprint submitted to Nucl. Instr. Meth. (2002).

10. Aglietta M. et al., Nucl. Instr. Meth. A277 (1989) 23.

11. Doll P. et al., Nucl. Instr. Meth. A488 (2002) 517.

12. Bertaina M. et al., $27^{\text {th }}$ ICRC, Hamburg, (2001) 792.

13. Drexlin G. et al. Nucl. Instr. Meth. A289 (1990) 490.

14. Antoni T. et al., Astropart. Physics 16 (2002) 245.

15. Nagano M. et al., J. Phys. G10 (1984) 1295.

16. Bird D.J. et al., Astrophys. J. 424 (1994) 494. 